Research Article

\title{
Dynamic Behaviors of a Class of High-Order Fuzzy Difference Equations
}

\author{
Lili Jia \\ Dianchi College of Yunnan University, Kunming, 650228, China \\ Correspondence should be addressed to Lili Jia; lilijiadianchi@163.com
}

Received 21 January 2020; Accepted 23 March 2020; Published 25 April 2020

Academic Editor: Basil K. Papadopoulos

Copyright (c) 2020 Lili Jia. This is an open access article distributed under the Creative Commons Attribution License, which permits unrestricted use, distribution, and reproduction in any medium, provided the original work is properly cited.

The purpose of this paper is to give the conditions for the existence and uniqueness of positive solutions and the asymptotic stability of equilibrium points for the following high-order fuzzy difference equation: $x_{n+1}=\left(A x_{n-1} x_{n-2}\right) /\left(B+\sum_{i=3}^{k} C_{i} x_{n-i}\right) n=$ $0,1,2, \ldots$, where $x_{n}$ is the sequence of positive fuzzy numbers and the parameters $A, B, C_{3}, C_{4}, \ldots, C_{k}$ and initial conditions $x_{0}, x_{-1}, x_{-2}, x_{-i}(i=3,4, \ldots, k)$ are positive fuzzy numbers. Besides, some numerical examples describing the fuzzy difference equation are given to illustrate the theoretical results.

\section{Introduction}

It is well known that difference equations are one of the most widely used equations in various subject areas. In particular, many phenomena in the real world often rely on the establishment and solution of mathematical models describing them. In the last twenty five years of the twentieth century, the study on the difference equation developed rapidly and occupied important positions in many applied analyses, such as demographic analysis, finance, and biology (see, e.g., [1-5] and the references therein). However, as the complexity of the modeling systems is increasing, the uncertainty factors in the model increases dramatically. Obviously, the fact that the ordinary difference equation model is used to describe many practical problems is incomplete. In view of this, a fuzzy difference system is a powerful tool to solve the mathematical model with fuzzy parameters and initial values in the real world, and it is an interesting and far-reaching research for modeling uncertainty and for processing vague or subjective information in a mathematical model.

In 1998, DeVault et al. [6] discussed the periodic character of solutions of the following nonlinear difference equation:

$$
x_{n+1}=-\frac{1}{x_{n}}+\frac{A}{x_{n-1}}, \quad n=0,1, \ldots,
$$

where $A>0$.

Papaschinopoulos and Schinas [7] researched the following difference equations:

$$
\begin{gathered}
x_{n+1}=A+\frac{y_{n}}{x_{n-p}}, \\
y_{n+1}=A+\frac{x_{n}}{y_{n-q}}, \\
n=0,1, \ldots,
\end{gathered}
$$

where $A, x_{-p}, x_{-p+1}, \ldots, x_{0}, y_{-q}, y_{-q+1}, \ldots, y_{0}$ are the positive numbers and $p$ and $q$ are the positive integers.

In $2014, \mathrm{He}$ et al. [8] studied the periodicity of the positive solutions for the following fuzzy max-difference equation:

$$
x_{n+1}=\max \left\{\frac{A_{n}}{x_{n-m}}, x_{n-k}\right\}, \quad n=0,1, \ldots
$$

where $k, m \in N^{*}, A_{n}$ is a periodic sequence of fuzzy numbers, and initial values $x_{0}, x_{-1}, \ldots, x_{-d}$ are the positive fuzzy numbers with $d=\{k, m\}$. 
Zhang et al. [9] investigated the boundedness, persistence, and asymptotic behavior of a positive fuzzy solution of the following third-order fuzzy difference equation using a generalization of division for fuzzy numbers:

$$
x_{n+1}=A+\frac{x_{n-1}}{x_{n-1} x_{n-2}}, \quad n=0,1,2, \ldots
$$

where $A, x_{0}, x_{-1}, x_{-2}$ are the positive fuzzy numbers.

These bright spots in the above papers deserve our learning. Moreover, in 2017, Khastan [10] discussed the following fuzzy difference equation:

$$
x_{n+1}-q=w x_{n}, \quad n=0,1, \ldots,
$$

where $\left\{x_{n}\right\}$ is a sequence of the positive fuzzy numbers and $x_{0}, q$, and $w$ are the positive fuzzy numbers. In this paper, the generalization of division for the fuzzy number is used to investigate the existence, uniqueness, and global behavior of the solution.

Wang and Wang [11] analyzed the following nonlinear difference equation:

$$
x_{n+1}=\frac{a x_{n-l} x_{n-k}}{b x_{n-s}+c x_{n-t}}, \quad n=0,1, \ldots,
$$

where the initial conditions $x_{0}, x_{1}, \ldots, x_{-r}$ are the positive real numbers, $l, k, s, t$ are the nonnegative integers, $r=\max \{l, k, s, t\}$, and $a, b, c$ are the positive constants. Further, in 2017, the author in [12] investigated the asymptotic behavior of the equilibrium points for the following fuzzy difference equation:

$$
x_{n+1}=\frac{A x_{n-l} x_{n-2}}{D+B x_{n-3}+C x_{n-4}}, \quad n=0,1,2, \ldots,
$$

where $\left\{x_{n}\right\}$ is a sequence of the positive fuzzy numbers and the parameters $A, B, C, D$ and initial conditions $x_{-4}, x_{-3}, x_{-2}, x_{-1}, x_{0}$ are the positive fuzzy numbers. Besides, some interesting results can be found in [13-18] and the references therein.

Based on the above valuable theoretical results, this paper studies the following high-order fuzzy difference equation:

$$
x_{n+1}=\frac{A x_{n-1} x_{n-2}}{B+\sum_{i=3}^{k} C_{i} x_{n-i}}, \quad n=0,1,2, \ldots,
$$

where $x_{n}$ is a sequence of the positive fuzzy numbers and the parameters $A, B, C_{3}, C_{4}, \ldots, C_{k}$ and initial conditions $x_{0}, x_{-1}, x_{-2}, x_{-i}(i=3,4, \ldots, k)$ are the positive fuzzy numbers. The purpose of this paper is to study the asymptotic behavior of the equilibrium point of the fuzzy difference equation. The main method is to convert the fuzzy difference equation into a rational difference equation according to the fuzzy number theory, and then the properties of the solutions of the fuzzy difference equations are obtained by studying the corresponding constant difference equations. In addition, the theoretical results are verified by numerical examples.

Remark 1. It is well known that difference equations look simple in form, but the properties of their solutions are very complex, especially the dependence on parameters and initial values. The main contribution and innovation of this paper are as follows: (1) based on the practical application, fuzzy parameters and initial values are introduced to the known models, and the new model can better describe the objective natural phenomenon. Obviously, the model in reference [12] is a special case of the model in this paper. (2) To study the new model, some new analytical methods and techniques that is different from those mentioned in the references are obtained. (3) In this paper, the research contents are more rich than the related references. Firstly, the existence and uniqueness of positive fuzzy solutions are proved. Secondly, the nonzero equilibrium points of the corresponding ordinary difference equations which are unstable are obtained by using the linearization method. Finally, it was found that the zero trivial solution of the fuzzy difference equation (8) is asymptotically stable when the parameters of the system are positive trivial fuzzy numbers. (4) The sufficient conditions obtained herein are new, general, and easily verifiable, which provide flexibility for the application and analysis of the high-order fuzzy difference equation.

\section{Preliminaries and Notations}

For the convenience of readers, the definitions and preliminary results related to the theoretical proof of the paper are given, see [19-23].

Definition 1. For a set $B, \bar{B}$ is denoted as the closure of $B$ and a function $A: R \longrightarrow[0,1]$ is a fuzzy number if the following conclusions are true:

(i) $A$ is normal, i.e., there exists $x \in R$ such that $A(x)=1$;

(ii) $A$ is a fuzzy convex set, i.e.,

$$
\begin{aligned}
& A\left(t x_{1}+(1-t) x_{2}\right) \geq \min \left\{A\left(x_{1}\right), A\left(x_{2}\right)\right\}, \\
& \forall t \in[0,1], x_{1}, x_{2} \in R
\end{aligned}
$$

(iii) $A$ is upper semicontinuous on $R$;

(iv) The $\frac{\text { support }}{A=\frac{\text { of }}{U_{\alpha \in(0,1]}[A]_{\alpha}}=\frac{A}{\{x \in R: A(x)>0\}} \text {, is compact, }}$ where the $\alpha$-cuts of $A$ are closed intervals, defined as $[A]_{\alpha}=\{x \in R: A(x) \geq \alpha\}$, and if supp $A \subset(0, \infty)$, then the fuzzy number $A$ is obviously positive.

Definition 2. Let $A$ and $B$ be the fuzzy numbers which satisfy $[A]_{\alpha}=\left[A_{l, \alpha}, A_{r, \alpha}\right]$ and $[B]_{\alpha}=\left[B_{l \alpha}, B_{r \alpha}\right], \alpha \in(0,1]$, then the following metric is denoted:

$$
D(A, B)=\sup \max \left\{\left|A_{l, \alpha}-B_{l, \alpha}\right|,\left|A_{r, \alpha}-B_{r, \alpha}\right|\right\},
$$

where sup is taken for all $\alpha \in(0,1]$. Then, $\left(R_{f}, D\right)$ is a complete metric space. For the convenience of application in the future, $\widehat{0} \in R_{f}$ is defined as 


$$
\widehat{0}(x)= \begin{cases}1, & x=0, \\ 0, & x \neq 0 .\end{cases}
$$

Thus, $[\widehat{0}]_{\alpha}=[0,0], \quad 0<\alpha \leq 1$.

Definition 3. Persistence (resp., boundedness) of fuzzy numbers is defined if there exists a positive real number $M$ (resp.N), such that the following conclusions are true: $\operatorname{supp} x_{n} \subset[M, \infty)\left(\operatorname{resp} . \operatorname{supp} x_{n} \subset(0, N]\right), n=1,2, \ldots$, where $\left\{x_{n}\right\}$ is a sequence of the positive fuzzy numbers.

Further, $\left\{x_{n}\right\}$ is bounded and persistent if there exist positive real numbers $M, N$, such that $\operatorname{supp} x_{n} \subset[M, N], n=1,2, \ldots$.

Lemma 1. Let $I_{x}, I_{y}$ be some intervals of real numbers and let $f: I_{x}^{k+1} \times I_{y}^{l+1} \longrightarrow I_{x}, g: I_{x}^{k+1} \times I_{y}^{l+1} \longrightarrow I_{y}$ be continuously differentiable functions. Then, for every set of initial conditions $\left(x_{i}, y_{j}\right) \in I_{x} \times I_{y}, \quad(i=-k,-k+1, \ldots, 0, j=-l$, $-l+1, \ldots, 0)$, the following system of difference equations:

$$
\left\{\begin{array}{l}
x_{n+1}=f\left(x_{n}, x_{n-1}, \ldots, x_{n-k}, y_{n}, y_{n-1}, \ldots, y_{n-l}\right), \\
y_{n+1}=g\left(x_{n}, x_{n-1}, \ldots, x_{n-k}, y_{n}, y_{n-1}, \ldots, y_{n-l}\right), \\
n=0,1,2, \ldots
\end{array}\right.
$$

has a unique solution $\left\{\left(x_{i}, y_{j}\right)\right\}_{i=-k, j=-l}^{+\infty,+\infty}$.

Definition 4. A point $(\bar{x}, \bar{y}) \in I_{x} \times I_{y}$ is called an equilibrium point of system (12) if $\bar{x}=f(\bar{x}, \bar{x}, \ldots, \bar{x}, \bar{y}$, $\bar{y}, \ldots, \bar{y}), \bar{y}=g(\bar{x}, \bar{x}, \ldots, \bar{x}, \bar{y}, \bar{y}, \ldots, \bar{y})$, that is, $\left(x_{n}, y_{n}\right)=$ $(\bar{x}, \bar{y})$ for $n \geq 0$ is the solution of difference system (12), or equivalently, $(\bar{x}, \bar{y})$ is a fixed point of the vector map $(f, g)$.

Definition 5. Suppose that $(\bar{x}, \bar{y})$ is an equilibrium point of system (12), then the following is obtained:

(i) $(\bar{x}, \bar{y})$ is called locally stable if for every $\varepsilon>0$, there exists $\delta>0$ such that for any initial conditions $\left(x_{i}, y_{i}\right) \in I_{x} \times I_{y}(i=-k, \ldots, 0, j=-l, \ldots, 0)$ with $\sum_{i=-k}^{0}\left|x_{i}-\bar{x}\right|<\delta, \sum_{j=-l}^{0}\left|y_{j}-\bar{y}\right|<\delta$, there is $s$ for any $n>0$;

(ii) $(\bar{x}, \bar{y})$ is called an attractor if $\lim _{n \longrightarrow \infty} x_{n}=\bar{x}$, $\lim _{n \longrightarrow \infty} y_{n}=\bar{y}$ for any initial conditions $\left(x_{i}, y_{i}\right) \in I_{x} \times I_{y}(i=-k, \ldots, 0, j=-l, \ldots, 0)$;

(iii) $(\bar{x}, \bar{y})$ is called asymptotically stable if it is stable and an attractor;

(iv) $(\bar{x}, \bar{y})$ is called unstable if it is not locally stable.

Definition 6. Let $(\bar{x}, \bar{y})$ be an equilibrium point of the vector $\operatorname{map} F=\left(f, x_{n}, \ldots, x_{n-k}, g, y_{n}, \ldots, y_{n-l}\right)$, where $f$ and $g$ are continuously differential functions at $(\bar{x}, \bar{y})$. The linearized system of (12) about the equilibrium point $(\bar{x}, \bar{y})$ is $X_{n+1}=F\left(X_{n}\right)=F_{j} \cdot X_{n}$, where $F_{J}$ is the Jacobian matrix of system (12) about $(\bar{x}, \bar{y})$ and $X_{n}=\left(x_{n}, \ldots, x_{n-k}\right.$, $\left.y_{n}, \ldots, y_{n-l}\right)^{T}$.

Definition 7. Let $p, q, s, t$ be four nonnegative integers such that $p+q=n$ and $s+t=m$. Split $x=\left(x_{1}, x_{2}, \ldots, x_{n}\right)$ into $x=\left([x]_{p},[x]_{q}\right)$ and $y=\left(y_{1}, y_{2}, \ldots, y_{m}\right)$ into $y=\left([y]_{s}\right.$, $\left.[y]_{t}\right)$, where $[x]_{\sigma}$ denotes a vector with $\sigma$-components of $x$. The function $f\left(x_{1}, x_{2}, \ldots, x_{n}, y_{1}, y_{2}, \ldots, y_{m}\right)$ possesses a mixed monotone property in subsets $I_{x}^{n} \times I_{y}^{m}$ of $R^{n} \times R^{m}$ if $f\left([x]_{p},[x]_{q},[y]_{s},[y]_{t}\right)$ is monotone nondecreasing in each component of $\left([x]_{q},[y]_{t}\right)$ for $(x, y) \in I_{x}^{n} \times I_{y}^{m}$. In particular, if $q=0, t=0$, then it is said to be monotone nondecreasing in $I_{x}^{n} \times I_{y}^{m}$.

Lemma 2. Assume that $X(n+1)=F(X(n)), n=0,1, \ldots$, is a system of difference equations and $\bar{X}$ is the equilibrium point of this system, i.e., $F(\bar{X})=\bar{X}$, then the following is obtained:

(i) If all eigenvalues of the Jacobian matrix $J_{F}$ about $\bar{X}$ lie inside the open unit disk $|\lambda|<1$, then $\bar{X}$ is locally asymptotically stable;

(ii) If one of the eigenvalues of the Jacobian $J_{F}$ matrix about $\bar{X}$ has a norm greater than one, then $\bar{X}$ is unstable.

Lemma 3. Assume that $X(n+1)=F(X(n)), n=0,1, \ldots$ is a system of difference equations and $\bar{X}$ is the equilibrium point of this system, and the characteristic polynomial of this system about the equilibrium point $\bar{X}$ is $P(\lambda)=a_{0}$ $\lambda^{n}+a_{1} \lambda^{n-1}+\cdots+a_{n-1} \lambda+a_{n}=0$, with the real coefficients and $a_{0}>0$. Then, all roots of the polynomial $P(\lambda)$ lie inside the open unit disk $|\lambda|<1$ if and only if

$$
\Delta_{k}>0, \quad \text { for } k=1,2, \ldots, n,
$$

where $\Delta_{k}$ is the principal minor of order $k$ of the $n \times n$ matrix:

$$
\Delta_{n}=\left[\begin{array}{ccccc}
a_{1} & a_{3} & a_{5} & \cdots & 0 \\
a_{0} & a_{2} & a_{4} & \cdots & 0 \\
0 & a_{1} & a_{3} & \cdots & 0 \\
\vdots & \vdots & \vdots & \ddots & \vdots \\
0 & 0 & 0 & \cdots & a_{n}
\end{array}\right] .
$$

\section{Main Results and Their Proofs}

The following lemmas are applied to study the existence and uniqueness of a positive solution of the fuzzy difference equation (8).

Lemma 4 (see [24]). Let $f$ be a continuous function from $R^{+} \times R^{+} \times R^{+}$into $R^{+}$and $A, B, C$ be the fuzzy numbers, then

$$
[f(A, B, C)]_{\alpha}=f\left([A]_{\alpha},[B]_{\alpha},[C]_{\alpha}\right), \quad \alpha \in(0,1] .
$$

Lemma 5 (see $[19,25]$ ). Let us denote $u \in R_{f}$ such that $[u]_{\alpha}=\left[u_{l, \alpha}, u_{r, \alpha}\right], \alpha \in(0,1]$, then $u_{l, \alpha}$ and $u_{r, \alpha}$ can be seen as functions on $\alpha \in(0,1]$ which satisfy the following conclusions:

(i) $u_{l, \alpha}$ is nondecreasing and left continuous;

(ii) $u_{r, \alpha}$ is nonincreasing and left continuous;

(iii) $u_{l, \alpha} \leq u_{r, \alpha}$. 
Conversely, for any functions $a(\alpha)$ and $b(\alpha)$ defined on $(0,1]$ which satisfy the above (i)-(iii), there exists a unique $u \in R_{f}$ such that $u(\alpha)=[a(\alpha), b(\alpha)]$ for any $\alpha \in(0,1]$.

Theorem 1. Consider equation (8), where $A, B, C_{i}(i=$ $3,4, \ldots, k)$ are the positive fuzzy numbers, and then for any positive fuzzy numbers $x_{0}, x_{-1}, x_{-2}, x_{-i}(i=3,4, \ldots, k)$, there exists a unique positive solution $\left\{x_{n}\right\}$ of (8) with the initial conditions $x_{0}, x_{-1}, x_{-2}, x_{-i}(i=3,4, \ldots, k)$.

Proof. Assume that there exists a sequence $\left\{x_{n}\right\}$ that is the positive solution of (8) with the positive parameters and initial conditions $A, B, C_{i}, x_{0}, x_{-1}, x_{-2}, x_{-i}(i=3,4, \ldots, k)$. Consider the $\alpha$-cuts, $\alpha \in(0,1]$,

$$
\begin{aligned}
{[A]_{\alpha}=} & {\left[A_{l, \alpha}, A_{r, \alpha}\right],[B]_{\alpha}=\left[B_{l, \alpha}, B_{r, \alpha}\right],\left[C_{i}\right]_{\alpha}=\left[C_{i l, \alpha}, C_{i r, \alpha}\right], } \\
& i=3,4, \ldots, k, \\
{\left[x_{n}\right]_{\alpha}=} & {\left[L_{n, \alpha}, R_{n, \alpha}\right], \quad n=-k,-k+1, \ldots, 0 . }
\end{aligned}
$$

From (8) and (16) and Lemma 4, the following is obtained:

$$
\begin{aligned}
{\left[x_{n+1}\right]_{\alpha} } & =\left[L_{n+1, \alpha}, R_{n+1, \alpha}\right]=\left[\frac{A x_{n-1} x_{n-2}}{B+\sum_{i=3}^{k} C_{i} x_{n-i}}\right]_{\alpha}=\frac{\left[A x_{n-1} x_{n-2}\right]_{\alpha}}{\left[B+\sum_{i=3}^{k} C_{i} x_{n-i}\right]_{\alpha}} \\
& =\frac{\left[A_{l, \alpha}, A_{r, \alpha}\right]\left[L_{n-1, \alpha}, R_{n-1, \alpha}\right]\left[L_{n-2, \alpha}, R_{n-2, \alpha}\right]}{\left[B_{l, \alpha}, B_{r, \alpha}\right]+\left[\sum_{i=3}^{k} C_{i l, \alpha}, \sum_{i=3}^{k} C_{i r, \alpha}\right]\left[\sum_{i=3}^{k} L_{n-i, \alpha}, \sum_{i=3}^{k} R_{n-i, \alpha}\right]} \\
& =\left[\frac{A_{l, \alpha} L_{n-1, \alpha} L_{n-2, \alpha}}{B_{r, \alpha}+\sum_{i=3}^{k} C_{i r, \alpha} R_{n-i, \alpha}}, \frac{A_{r, \alpha} R_{n-1, \alpha} R_{n-2, \alpha}}{B_{l, \alpha}+\sum_{i=3}^{k} C_{i l, \alpha} L_{n-i, \alpha}}\right] .
\end{aligned}
$$

Hence, for $\alpha \in(0,1], n=-k,-k+1, \ldots, 0$, according to the above result, it follows that

$$
\begin{aligned}
L_{n+1, \alpha} & =\frac{A_{l, \alpha} L_{n-1, \alpha} L_{n-2, \alpha}}{B_{r, \alpha}+\sum_{i=3}^{k} C_{i r, \alpha} R_{n-i, \alpha}}, \\
R_{n+1, \alpha} & =\frac{A_{r, \alpha} R_{n-1, \alpha} R_{n-2, \alpha}}{B_{l, \alpha}+\sum_{i=3}^{k} C_{i l, \alpha} L_{n-i, \alpha}} .
\end{aligned}
$$

And then, for any initial conditions $\left(L_{j, \alpha}, R_{j, \alpha}\right), j=-k,-k+1, \ldots, 0, \alpha \in(0,1], \quad$ it is evident from Lemma 1 that there exists a unique solution $\left(L_{n, \alpha}, R_{n, \alpha}\right)$ of the ordinary difference equations (18).

Conversely, it is proved that the positive solution $\left\{x_{n}\right\}$ of equation (8) is determined by $\left(L_{n, \alpha}, R_{n, \alpha}\right)$ with initial conditions $\left(L_{j, \alpha}, R_{j, \alpha}\right), j=-k,-k+1, \ldots, 0, \alpha \in(0,1]$ and satisfies the following condition:

$$
\left[x_{n}\right]_{\alpha}=\left[L_{n, \alpha}, R_{n, \alpha}\right], \quad \alpha \in(0,1], n=-k,-k+1, \ldots
$$

For any $\alpha_{1}, \alpha_{2} \in(0,1], \alpha_{1}<\alpha_{2}$, according to Lemma 5 , the following is obtained:

$$
\begin{aligned}
0< & A_{l, \alpha_{1}} \leq A_{l, \alpha_{2}} \leq A_{r, \alpha_{2}} \leq A_{r, \alpha_{1}}, 0<C_{i l, \alpha_{1}} \leq C_{i l, \alpha_{2}} \leq C_{i r, \alpha_{2}} \leq C_{i r, \alpha_{1}}, \\
& \quad i=3,4, \ldots, k, \\
0< & B_{l, \alpha_{1}} \leq B_{l, \alpha_{2}} \leq B_{r, \alpha_{2}} \leq B_{r, \alpha_{1}}, 0<L_{j, \alpha_{1}} \leq L_{j, \alpha_{2}} \leq R_{j, \alpha_{2}} \leq R_{j, \alpha_{1}}, \\
& \quad j=-k,-k+1, \ldots, 0,
\end{aligned}
$$

where $A, B, C_{i}, x_{j}(i=3,4, \ldots, k, j=-k,-k+1, \ldots, 0)$ are the positive fuzzy numbers.

Next, from the mathematical induction, it is proved that the following conclusion is true, that is,

$$
0<L_{n, \alpha_{1}} \leq L_{n, \alpha_{2}} \leq R_{n, \alpha_{2}} \leq R_{n, \alpha_{1}}, \quad n=1,2, \ldots
$$

According to (20), (21) is true for $n=-k,-k+1, \ldots, 0$. Suppose that (21) is true for any $n \leq m, m \in\{1,2, \ldots\}$, then from (18)-(21), it follows that for $n=m+1$,

$$
\begin{aligned}
& L_{m+1, \alpha_{1}}=\frac{A_{l, \alpha_{1}} L_{m-1, \alpha_{1}} L_{m-2, \alpha_{1}}}{B_{r, \alpha_{1}}+\sum_{i=3}^{k} C_{i r, \alpha_{1}} R_{m-i, \alpha_{1}}} \leq \frac{A_{l, \alpha_{2}} L_{m-1, \alpha_{2}} L_{m-2, \alpha_{2}}}{B_{r, \alpha_{2}}+\sum_{i=3}^{k} C_{i r, \alpha_{2}} R_{m-i, \alpha_{2}}}=L_{m+1, \alpha_{2}}, \\
& L_{m+1, \alpha_{2}} \leq \frac{A_{r, \alpha_{2}} R_{m-1, \alpha_{2}} R_{m-2, \alpha_{2}}}{B_{l, \alpha_{2}}+\sum_{i=3}^{k} C_{i l, \alpha_{2}} L_{m-i, \alpha_{2}}}=R_{m+1, \alpha_{2}} \leq \frac{A_{r, \alpha_{1}} R_{m-1, \alpha_{1}} R_{m-2, \alpha_{1}}}{B_{l, \alpha_{1}}+\sum_{i=3}^{k} C_{i l, \alpha_{1}} L_{m-i, \alpha_{1}}}=R_{m+1, \alpha_{1}} .
\end{aligned}
$$


Hence, (21) is true.

Furthermore, from (18), it follows that

$$
\begin{aligned}
& L_{1, \alpha}=\frac{A_{l, \alpha} L_{-1, \alpha} L_{-2, \alpha}}{B_{r, \alpha}+\sum_{i=3}^{k} C_{i r, \alpha} R_{-i, \alpha}}, \\
& R_{1, \alpha}=\frac{A_{r, \alpha} R_{-1, \alpha} R_{-2, \alpha}}{B_{l, \alpha}+\sum_{i=3}^{k} C_{i l, \alpha} L_{-i, \alpha}}, \\
& \alpha \in(0,1] .
\end{aligned}
$$

Then, in view of $A, B, C_{i}, x_{j}(i=3,4, \ldots, k, j=-k,-k+$ $1, \ldots, 0)$ which are the positive fuzzy numbers, it is known that $A_{l, \alpha}, A_{r, \alpha}, B_{l, \alpha}, B_{r, \alpha}, C_{i l, \alpha}, C_{i r, \alpha}, L_{-k, \alpha}, R_{-k, \alpha}, L_{-k+1, \alpha}, R_{-k+1,}$ $\alpha, \ldots, L_{0, \alpha}, R_{0, \alpha},(i=3,4, \ldots, k)$ are left continuous from Lemma 5. So, $L_{1, \alpha}$ and $R_{1, \alpha}$ are also left continuous from (23). Moreover, $L_{n, \alpha}, R_{n, \alpha}, n=1,2, \ldots$ are left continuous using mathematical induction.
Now, it is proven that $x_{n}$ is supported, i.e., supp $x_{n}=$ $\overline{\mathrm{U}_{\alpha \in(0,1]}\left[L_{n, \alpha}, R_{n, \alpha}\right]}$ is compact. It is easy to know that it is enough to prove that $\cup_{\alpha \in(0,1]}\left[L_{n, \alpha}, R_{n, \alpha}\right]$ is bounded.

Let $n=1$, from $A, B, C_{i}, x_{j}(i=3,4, \ldots, k, j=-k,-k+$ $1, \ldots, 0)$ which are the positive fuzzy numbers, then there exist constants $M_{i}, N_{i}>0, i=3,4, \ldots, k$, such that for all $\alpha \in(0,1]$,

$$
\begin{aligned}
{\left[A_{l, \alpha}, A_{r, \alpha}\right] } & \subset\left[M_{1}, N_{1}\right], \\
{\left[B_{l, \alpha}, B_{r, \alpha}\right] } & \subset\left[M_{2}, N_{2}\right], \\
{\left[C_{i l, \alpha}, C_{i r, \alpha}\right] } & \subset\left[M_{1}, N_{1}\right], \quad i=3,4, \ldots, k, \\
{\left[L_{j, \alpha}, R_{j, \alpha}\right] } & \subset\left[M_{1}, N_{1}\right], \quad j=-k,-k+1, \ldots, 0 .
\end{aligned}
$$

Therefore, relations (23) and (24) imply that

$$
\left[L_{1, \alpha}, R_{1, \alpha}\right] \subset\left[\frac{M_{1} M_{-1} M_{-2}}{N_{2}+\sum_{i=3}^{k} \sum_{j=-3}^{-k} N_{i} N_{j}}, \frac{N_{1} N_{-1} N_{-2}}{M_{2}+\sum_{i=3}^{k} \sum_{j=-3}^{-k} M_{i} M_{j}}\right], \quad \alpha \in(0,1]
$$

from which the following is obtained:

$$
\bigcup_{\alpha \in(0,1]}\left[L_{1, \alpha}, R_{1, \alpha}\right] \subset\left[\frac{M_{1} M_{-1} M_{-2}}{N_{2}+\sum_{i=3}^{k} \sum_{j=-3}^{-k} N_{i} N_{j}}, \frac{N_{1} N_{-1} N_{-2}}{M_{2}+\sum_{i=3}^{k} \sum_{j=-3}^{-k} M_{i} M_{j}}\right], \quad \alpha \in(0,1] .
$$

From (26), $\overline{\mathrm{U}_{\alpha \in(0,1]}\left[L_{1, \alpha}, R_{1, \alpha}\right]}$ is compact and $\overline{\mathrm{U}_{\alpha \in(0,1]}\left[L_{1, \alpha}, R_{1, \alpha}\right]} \subset(0, \infty)$. Moreover, under the mathematical induction method, it is also proven that $\mathrm{U}_{\alpha \in(0,1]}\left[L_{1, \alpha}, R_{1, \alpha}\right]$ is compact and

$$
\overline{\cup_{\alpha \in(0,1]}\left[L_{1, \alpha}, R_{1, \alpha}\right]} \subset(0, \infty), \quad n=1,2, \ldots
$$

Since (21) and (24) and $L_{n, \alpha}, R_{n, \alpha}$ are left continuous, from Lemma 5 , a sequence of positive fuzzy numbers $\left\{x_{n}\right\}$ is determined $\left[L_{n, \alpha}, R_{n, \alpha}\right]$ such that (8) holds.

Furthermore, it is proven that $\left\{x_{n}\right\}$ is the solution of (6) with initial conditions $x_{0}, x_{-1}, x_{-2}, x_{-i}(i=3,4, \ldots, k)$. Since for all $\alpha \in(0,1]$,

$$
\left[x_{n+1}\right]_{\alpha}=\left[L_{n+1, \alpha}, R_{n+1, \alpha}\right]=\left[\frac{A_{l, \alpha} L_{n-1, \alpha} L_{n-2, \alpha}}{B_{r, \alpha}+\sum_{i=3}^{k} C_{i r, \alpha} R_{n-i, \alpha}}, \frac{A_{r, \alpha} R_{n-1, \alpha} R_{n-2, \alpha}}{B_{l, \alpha}+\sum_{i=3}^{k} C_{i r, \alpha} L_{n-i, \alpha}}\right]=\left[\frac{A x_{n-1} x_{n-2}}{B+\sum_{i=3}^{k} C_{i} x_{n-i}}\right]_{\alpha}
$$

it is claimed that $\left\{x_{n}\right\}$ is the solution of (6) with initial conditions $x_{0}, x_{-1}, x_{-2}, x_{-i},(i=3,4, \ldots, k)$.

Now, its uniqueness by contradiction is proven in the following. Assume that there exists another positive solution $\left\{x_{n}^{*}\right\}$ of (8) with initial conditions $x_{0}, x_{-1}$, $x_{-2}, x_{-i}(i=3,4, \ldots, k)$, and according to a similar discussion above, it holds that

$$
\left[x_{n}^{*}\right]_{\alpha}=\left[L_{n, \alpha}, R_{n, \alpha}\right], \quad \alpha \in(0,1], n=0,1, \ldots,
$$

and then, from (19) and (29), one has that

$$
\left[x_{n}\right]_{\alpha}=\left[x_{n}^{*}\right]_{\alpha}, \quad \alpha \in(0,1], n=-k,-k+1, \ldots,
$$

and from the above result, it is obtained that the sequence $\left\{x_{n}\right\}$ is a unique solution of (6) with initial conditions $x_{0}, x_{-1}, x_{-2}, x_{-i}(i=3,4, \ldots, k)$, so the proof is completed.

In the following result, the asymptotic behavior of the equilibrium point of (6) is further investigated. From the above proof process, it is known that if $x_{n}$ is the unique positive solution of (6) with the initial values $x_{0}, x_{-1}, x_{-2}, x_{-i}(i=3,4, \ldots, k)$, then the following is obtained:

$$
\left[x_{n}\right]_{\alpha}=\left[L_{n, \alpha}, R_{n, \alpha}\right], \alpha \in(0,1], \quad n=0,1, \ldots
$$


Hence, it is known that $\left(L_{n, \alpha}, R_{n, \alpha}\right)$ satisfies the family of system (18). The following corresponding ordinary parametric systems to study the asymptotic behavior of equation (8) are constructed:

$$
\begin{aligned}
y_{n+1}= & \frac{a_{1} y_{n-1} y_{n-2}}{b_{1}+\sum_{i=3}^{k} c_{1 i} z_{n-i}}, \\
z_{n+1}=\frac{a_{2} z_{n-1} z_{n-2}}{b_{2}+\sum_{i=3}^{k} c_{2 i} y_{n-i}}, & \\
n & =0,1, \ldots,
\end{aligned}
$$

where the parameters $a_{1}, a_{2}, b_{1}, b_{2}, c_{1 i}, c_{2 i}(i=3,4, \ldots, k)$ and initial conditions $y_{0}, y_{-1}, y_{-2}, y_{-i}, z_{0}, z_{-1}, z_{-2}, z_{-i}(i=3,4, \ldots, k)$ are the positive real constants. Clearly, system (32) has a unique solution $\left(y_{n}, z_{n}\right)$ for any initial conditions from Lemma 1.

Now, it is easily obtained that system (32) has the following three equilibrium points:

$$
\begin{aligned}
& \bar{X}_{1}=\left(\bar{y}_{1}, \bar{z}_{1}\right)=(0,0), \\
& \bar{X}_{2}=\left(\bar{y}_{2}, \bar{z}_{2}\right)=\left(0, \frac{b_{2}}{a_{2}}\right), \\
& \bar{X}_{3}=\left(\bar{y}_{3}, \bar{z}_{3}\right)=\left(\frac{b_{1}}{a_{1}}, 0\right),
\end{aligned}
$$

if $a_{1} a_{2}>\sum_{i=3}^{k} c_{1 i} \sum_{i=3}^{k} c_{2 i}$, and system (32) has the fourth positive equilibrium point $\bar{X}_{4}$ :

$$
\bar{X}_{4}=\left(\bar{y}_{4}, \bar{z}_{4}\right)=\left(\frac{a_{2} b_{1}+b_{2} \sum_{i=3}^{k} c_{1 i}}{a_{1} a_{2}-\sum_{i=3}^{k} c_{1 i} \sum_{i=3}^{k} c_{2 i}}, \frac{a_{1} b_{2}+b_{1} \sum_{i=3}^{k} c_{2 i}}{a_{1} a_{2}-\sum_{i=3}^{k} c_{1 i} \sum_{i=3}^{k} c_{2 i}}\right) .
$$

Next, the asymptotic behavior of these four equilibrium points in detail is analyzed.

Theorem 2. The equilibrium point $\bar{X}_{1}=(0,0)$ is locally asymptotically stable.

Proof. Let $F:\left(R^{+}\right)^{k} \longrightarrow R^{+}, G:\left(R^{+}\right)^{k} \longrightarrow R^{+}$be multivariate functions defined by

$$
\begin{aligned}
F\left(y_{n-1}, y_{n-2}, z_{n-3}, \ldots, z_{n-k}\right) & =\frac{a_{1} y_{n-1} y_{n-2}}{b_{1}+\sum_{i=3}^{k} c_{1 i} z_{n-i}}, \\
G\left(z_{n-1}, z_{n-2}, y_{n-3}, \ldots, y_{n-k}\right) & =\frac{a_{2} z_{n-1} z_{n-2}}{b_{2}+\sum_{i=3}^{k} c_{2 i} y_{n-i}}, \\
n & =0,1, \ldots,
\end{aligned}
$$

thus, it holds that

$$
\begin{aligned}
& F_{y_{n-1}}=\frac{a_{1} y_{n-2}}{b_{1}+\sum_{i=3}^{k} c_{1 i} z_{n-i}}, \\
& F_{y_{n-2}}=\frac{a_{1} y_{n-1}}{b_{1}+\sum_{i=3}^{k} c_{1 i} z_{n-i}}, \\
& F_{z_{n-i}}=\frac{-a_{1} c_{1 i} y_{n-1} y_{n-2}}{\left(b_{1}+\sum_{i=3}^{k} c_{1 i} z_{n-i}\right)^{2}} \\
& G_{z_{n-1}}=\frac{a_{2} z_{n-2}}{b_{2}+\sum_{i=3}^{k} c_{2 i} y_{n-i}}, \\
& G_{z_{n-2}}=\frac{a_{2} z_{n-1}}{b_{2}+\sum_{i=3}^{k} c_{2 i} y_{n-i}}, \\
& G_{y_{n-i}}=-\frac{a_{2} c_{2 i} z_{n-1} z_{n-2}}{\left(b_{2}+\sum_{i=3}^{k} c_{2 i} y_{n-i}\right)^{2}} .
\end{aligned}
$$

Moreover, the linearized system of (32) about the equilibrium point $\bar{X}_{1}$ is provided by

$$
\varphi_{n+1}=D_{1} \varphi_{n}
$$

where

$$
\varphi_{n}=\left[\begin{array}{c}
y_{n} \\
y_{n-1} \\
\vdots \\
y_{n-k} \\
z_{n} \\
z_{n-1} \\
\vdots \\
z_{n-k}
\end{array}\right]
$$

$$
D_{1}=\left[\begin{array}{ccccccccc}
0 & 0 & \cdots & 0 & 0 & 0 & \cdots & 0 & 0 \\
1 & 0 & \cdots & 0 & 0 & 0 & \cdots & 0 & 0 \\
\cdots & \cdots & \cdots & \cdots & \cdots & \cdots & \cdots & \cdots & \cdots \\
0 & 0 & \cdots & 1 & 0 & 0 & \cdots & 0 & 0 \\
0 & 0 & \cdots & 0 & 0 & 0 & \cdots & 0 & 0 \\
0 & 0 & \cdots & 0 & 0 & 1 & \cdots & 0 & 0 \\
\cdots & \cdots & \cdots & \cdots & \cdots & \cdots & \cdots & \cdots & \cdots \\
0 & 0 & \cdots & 0 & 0 & 0 & \cdots & 1 & 0
\end{array}\right],
$$

and the corresponding characteristic polynomial of $D_{1}(0,0)$ is as follows:

$$
f(\lambda)=\lambda^{2(k+1)}=0 .
$$

Obviously, all eigenvalues of $D_{1}(0,0)$ about $(0,0)$ lie in an open unit disk $|\lambda|<1$; hence, from Lemma 2, the 
equilibrium point $\bar{X}_{1}$ is locally asymptotically stable, and then the proof ends.

Theorem 3. The equilibrium point $\bar{X}_{2}$ is unstable.

Proof. From (36), the linearized systems of (32) about the equilibrium point $\bar{X}_{2}$ is as follows:

$$
\varphi_{n+1}=D_{2} \varphi_{n}
$$

where

$$
\varphi_{n}=\left[\begin{array}{c}
y_{n} \\
y_{n-1} \\
y_{n-2} \\
\vdots \\
y_{n-k} \\
z_{n} \\
z_{n-1} \\
\vdots \\
z_{n-k}
\end{array}\right],
$$

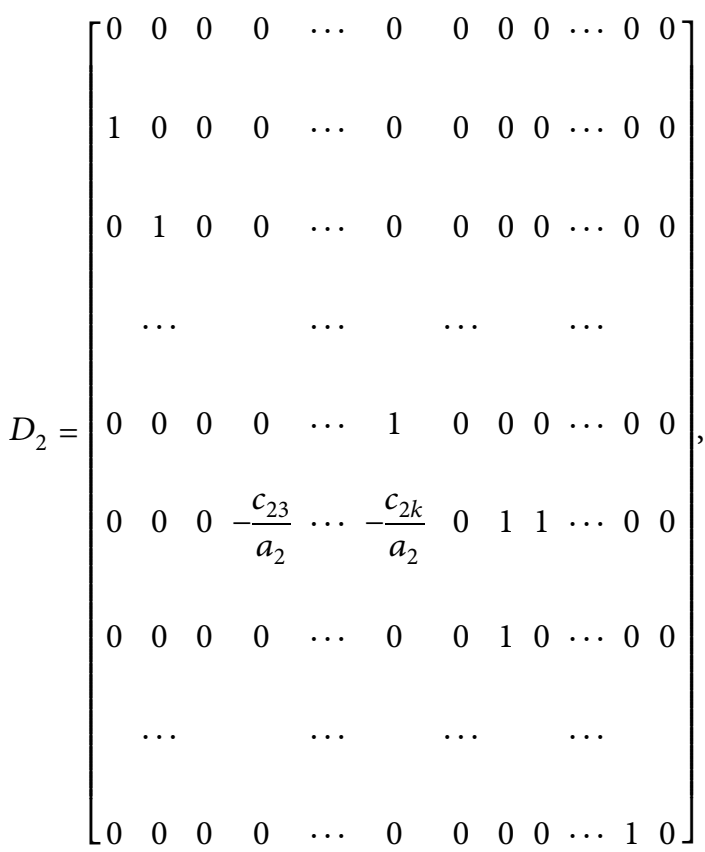

and the characteristic polynomial of (40) is given by

$$
f(\lambda)=\lambda^{2 k-1}\left(\lambda^{3}-\lambda-1\right)=0 .
$$

It is obvious that there exists $|\lambda|>1$ so that $\lambda^{2 k-1}\left(\lambda^{3}-\right.$ $\lambda-1)=0$. Thus, a root of the characteristic equation (42) lies outside the unit disk. According to Lemma 2, the equilibrium point $\bar{X}_{2}$ of (32) is unstable, and the proof is complete.
Theorem 4. The equilibrium point $\bar{X}_{3}$ is unstable.

Proof. From (36), the linearized systems of (36) about the equilibrium point $\bar{X}_{3}$ is provided by

$$
\varphi_{n+1}=D_{3} \varphi_{n}
$$

where

$$
\varphi_{n}=\left[\begin{array}{cccccccccc}
y_{n} \\
y_{n-1} \\
y_{n-2} \\
\vdots
\end{array}\right]
$$

and the characteristic polynomial of (43) is given by

$$
f(\lambda)=\lambda^{2 k-1}\left(\lambda^{3}-\lambda-1\right)=0,
$$

which is the same as with (42); therefore, the equilibrium point $\bar{X}_{3}$ of (32) is unstable,, and the proof is complete. 
Theorem 5. If $a_{1} a_{2}>\sum_{i=3}^{k} c_{1 i} \sum_{i=3}^{k} c_{2 i}$, then the equilibrium point $\bar{X}_{4}$ is unstable.

$$
\varphi_{n+1}=D_{4} \varphi_{n}
$$

Proof. From (36), the linearized systems of (32) about the where equilibrium point $\bar{X}_{4}$ is provided by

$$
\begin{aligned}
& \varphi_{n}=\left[\begin{array}{c}
y_{n} \\
y_{n-1} \\
y_{n-2} \\
\vdots \\
y_{n-k} \\
z_{n} \\
z_{n-1} \\
\vdots \\
z_{n-k}
\end{array}\right], \\
& D_{4}=\left[\begin{array}{cccccccccccc}
0 & 1 & 1 & 0 & \cdots & 0 & 0 & 0 & -\frac{c_{13}}{a_{1}} & \cdots & -\frac{c_{1 k-1}}{a_{1}} & -\frac{c_{1 k}}{a_{1}} \\
1 & 0 & 0 & 0 & \cdots & 0 & 0 & 0 & 0 & \cdots & 0 & 0 \\
0 & 1 & 0 & 0 & \cdots & 0 & 0 & 0 & 0 & \cdots & 0 & 0 \\
& \cdots & & & \cdots & & \cdots & & \cdots & & \\
0 & 0 & 0 & 0 & \cdots & 1 & 0 & 0 & 0 & \cdots & 0 & 0 \\
0 & 0 & 0 & -\frac{c_{23}}{a_{2}} & \cdots & -\frac{c_{2 k}}{a_{2}} & 0 & 1 & 1 & \cdots & 0 & 0 \\
0 & 0 & 0 & 0 & \cdots & 0 & 0 & 1 & 0 & \cdots & 0 & 0 \\
0 & 0 & 0 & 0 & \cdots & 0 & 0 & 0 & 0 & \cdots & 1 & 0
\end{array}\right] \text {, }
\end{aligned}
$$

and the characteristic polynomial of (46) is given by

$$
f(\lambda)=\lambda^{2 k+2}-2 \lambda^{2 k}-2 \lambda^{2 k-1}+\lambda^{2 k-2}+2 \lambda^{2 k-3}+\lambda^{2 k-4}-\frac{1}{a_{1} a_{2}} \sum_{i=3}^{i=2 k-3} \sum_{j=3}^{j=2 k-3} c_{1 i} c_{2 j} \lambda^{2 k-i-j}=0 .
$$


According to Lemma 3, the following is obtained:

$$
\begin{aligned}
& \Delta_{1}=\left(a_{1}\right)=0 ; \\
& \Delta_{2}=\left(\begin{array}{cc}
0 & -2 \\
1 & -2
\end{array}\right)=2>0 \\
& \Delta_{3}=\left(\begin{array}{ccc}
0 & -2 & 2 \\
1 & -2 & 1 \\
0 & 0 & -2
\end{array}\right)=4>0 ; \\
& \Delta_{4}=\left(\begin{array}{cccc}
0 & -2 & 2 & 0 \\
1 & -2 & 1 & 1 \\
0 & 0 & -2 & 2 \\
0 & 0 & -2 & 1
\end{array}\right)=-4<0 .
\end{aligned}
$$

It is obvious that not all $\Delta_{i}>0, i=1,2, \ldots, 2 k+2$. From Lemma 2 , the equilibrium point $\bar{X}_{4}$ is unstable, and then the proof is completed.

Lemma 6 (see [12]). Let $I_{x}, I_{y}$ be some intervals of real numbers and assume that $f: I_{x}^{k+1} \times I_{y}^{l+1} \longrightarrow I_{x}$ and $g: I_{x}^{k+1} \times$ $I_{y}^{l+1} \longrightarrow I_{y}$ be continuously differentiable functions satisfying a mixed monotone property. If there exists

$$
\left\{\begin{array}{l}
m_{0} \leq \min \left\{x_{-k}, \ldots, x_{0}, y_{-l}, \ldots, y_{0}\right\} \leq \max \left\{x_{-k}, \ldots, x_{0}, y_{-l}, \ldots, y_{0}\right\} \leq M_{0} \\
n_{0} \leq \min \left\{x_{-k}, \ldots, x_{0}, y_{-l}, \ldots, y_{0}\right\} \leq \max \left\{x_{-k}, \ldots, x_{0}, y_{-l}, \ldots, y_{0}\right\} \leq N_{0}
\end{array}\right.
$$

such that

$$
\left\{\begin{array}{l}
m_{0} \leq f\left(\left[m_{0}\right]_{p},\left[M_{0}\right]_{q},\left[n_{0}\right]_{s},\left[N_{0}\right]_{t}\right) \leq f\left(\left[M_{0}\right]_{p},\left[m_{0}\right]_{q},\left[N_{0}\right]_{s},\left[n_{0}\right]_{t}\right) \leq M_{0}, \\
n_{0} \leq g\left(\left[m_{0}\right]_{p_{1}},\left[M_{0}\right]_{q_{1}},\left[n_{0}\right]_{s_{1}},\left[N_{0}\right]_{t_{1}}\right) \leq g\left(\left[M_{0}\right]_{p_{1}},\left[m_{0}\right]_{q_{1}},\left[N_{0}\right]_{s_{1}},\left[n_{0}\right]_{t_{1}}\right) \leq N_{0},
\end{array}\right.
$$

then there exist $(m, M) \in\left[m_{0}, M_{0}\right]^{2}$ and $(n, N) \in\left[n_{0}, N_{0}\right]^{2}$ satisfying

$$
\left\{\begin{array}{l}
M=f\left([M]_{p},[m]_{q},[N]_{s},[n]_{t}\right), m=f\left([m]_{p},[M]_{q},[n]_{s},[N]_{t}\right) \\
N=g\left([M]_{p_{1}},[m]_{q_{1}},[N]_{s_{1}},[n]_{t_{1}}\right), n=g\left([m]_{p_{1}},[M]_{q_{1}},[n]_{s_{1}},[N]_{t_{1}}\right) .
\end{array}\right.
$$

Moreover, if $m=M$ and $n=N$, then equation (12) has a unique equilibrium point $(\bar{x}, \bar{y}) \in\left[m_{0}, M_{0}\right] \times\left[n_{0}, N_{0}\right]$ and every solution of $(12)$ converges to $(\bar{x}, \bar{y})$.

Theorem 6. If $a_{1}=a_{2}, b_{1}=b_{2}, c_{1 i}=c_{2 i}$, then the equilibrium point $\bar{X}_{1}=(0,0)$ of system (32) is a global attractor for any conditions $\left(y_{-i}, z_{-i}\right) \in\left(0,\left(b_{1} / 2 a_{1}\right)\right) \times\left(0,\left(b_{1} / 2 a_{1}\right)\right), i=$ $-k,-k+1, \ldots, 0$.
Proof. In view of $a_{1}=a_{2}, b_{1}=b_{2}, c_{1 i}=c_{2 i}$, system (32) can be changed to

$$
\begin{aligned}
y_{n+1} & =\frac{a_{1} y_{n-1} y_{n-2}}{b_{1}+\sum_{i=3}^{k} c_{1 i} z_{n-i}}, \\
z_{n+1} & =\frac{a_{1} z_{n-1} z_{n-2}}{b_{1}+\sum_{i=3}^{k} c_{1 i} y_{n-i}}, \\
n & =0,1, \ldots
\end{aligned}
$$


Let $(f, g):\left(0,\left(b_{1} / 2 a_{1}\right)\right)^{2 k+2} \times\left(0,\left(b_{1} / 2 a_{1}\right)\right)^{2 k+2} \longrightarrow$ $(0, \infty) \times(0, \infty)$ be a function defined by

$$
\begin{aligned}
& f\left(y_{n}, y_{n-1}, \ldots, y_{n-i}, z_{n}, z_{n-1}, \ldots, z_{n-i}\right)=\frac{a_{1} y_{n-1} y_{n-2}}{b_{1}+\sum_{i=3}^{k} c_{1 i} z_{n-i}}, \\
& g\left(y_{n}, y_{n-1}, \ldots, y_{n-i}, z_{n}, z_{n-1}, \ldots, z_{n-i}\right)=\frac{a_{1} z_{n-1} z_{n-2}}{b_{1}+\sum_{i=3}^{k} c_{1 i} y_{n-i}} .
\end{aligned}
$$

Set

$$
\begin{aligned}
f & =\frac{a_{1} u v}{b_{1}+\sum_{i=3}^{k} c_{1 i} w_{i}}, \\
g & =\frac{a_{1} u^{*} v^{*}}{b_{1}+\sum_{i=3}^{k} c_{1 i} w_{i}^{*}}, \\
f_{u} & =\frac{a_{1} v}{b_{1}+\sum_{i=3}^{k} c_{1 i} w_{i}}>0, \\
f_{v} & =\frac{a_{1} u}{b_{1}+\sum_{i=3}^{k} c_{1 i} w_{i}}>0, \\
f_{w_{i}} & =-\frac{a_{1} c_{1 i} u v}{\left(b_{1}+\sum_{i=3}^{k} c_{1 i} w_{i}\right)^{2}}<0, \\
g_{u^{*}} & =\frac{a_{1} v^{*}}{b_{1}+\sum_{i=3}^{k} c_{1 i} w_{i}^{*}}>0, \\
g_{w_{i}^{*}} & =-\frac{a_{1} c_{1 i} u^{*} v^{*}}{\left(b_{1}+\sum_{i=3}^{k} c_{1 i} w_{i}\right)^{2}}<0, \\
g_{v^{*}} & =\frac{a_{1}+\sum_{i=3}^{k} c_{1 i} w_{i}^{*}}{b_{1}}>0,
\end{aligned}
$$

which means that $f$ and $g$ have a mixed monotone property.

Let

$$
\begin{gathered}
M_{0}=N_{0}=\max \left\{y_{0}, y_{-1}, y_{-2}, y_{-i}, z_{0}, z_{-1}, z_{-2}, z_{-i}\right\}, \\
i=3,4, \ldots, k, \\
{\left[\frac{\left(a_{1} M_{0}-b_{1}\right)}{\sum_{i=3}^{k} c_{1 i}}\right] \leq m_{0}=n_{0}<0,}
\end{gathered}
$$

then,

$$
\begin{aligned}
& m_{0} \leq \frac{a_{1} m_{0}^{2}}{b_{1}+N_{0} \sum_{i=3}^{k} c_{1 i}} \leq \frac{a_{1} M_{0}^{2}}{b_{1}+n_{0} \sum_{i=3}^{k} c_{1 i}} \leq M_{0}, \\
& n_{0} \leq \frac{a_{1} n_{0}^{2}}{b_{1}+M_{0} \sum_{i=3}^{k} c_{1 i}} \leq \frac{a_{1} N_{0}^{2}}{b_{1}+m_{0} \sum_{i=3}^{k} c_{1 i}} \leq N_{0} .
\end{aligned}
$$

Obviously, since $m_{i}=n_{i}$ and $M_{i}=N_{i}, i=0,1, \ldots$, then from system (32) and Lemma 6, there exist $m, M \in\left[m_{0}, M_{0}\right], n=m, N=M$ such that

$$
\begin{aligned}
& m=\frac{a_{1} m^{2}}{b_{1}+N \sum_{i=3}^{k} c_{1 i}}, \\
& n=\frac{a_{1} n^{2}}{b_{1}+M \sum_{i=3}^{k} c_{1 i}}, \\
& M=\frac{a_{1} M^{2}}{b_{1}+n \sum_{i=3}^{k} c_{1 i}}, \\
& N=\frac{a_{1} N^{2}}{b_{1}+m \sum_{i=3}^{k} c_{1 i}} .
\end{aligned}
$$

Thus, $\left[a_{1}(m+M)-b_{1}\right](m-M)=0$. Moreover, from $2 a_{1} M_{0}<b_{1}$, then $M=m$ and $N=n$.

Hence, it is obtained that the equilibrium point $(0,0)$ of system (32) is a global attractor from Lemma 6 . The proof is completed.

Moreover, from Definition 5, it is obtained that the equilibrium point $\bar{X}_{1}$ of system (32) is asymptotically stable.

It is obvious that the fuzzy difference (6) has the trivial solution $\widehat{0}$. Next, the stability of the trivial solution is discussed. To do this, first, the following definition is introduced.

Definition 8 . The trivial solution $x=\widehat{0}$ of the fuzzy difference equation (8) is said to be

(i) Stable, if given $\varepsilon>0$, and there exists $\delta(\varepsilon)>0$ with $D\left(x_{i}, \widehat{0}\right)<\delta, i=-k,-k+1, \ldots, 0$, which implies $D\left(x_{n}, \widehat{0}\right)<\varepsilon$, for any $n>0$, such that for any $x_{i} \in D_{\delta}, i=-k,-k+1, \cdots, 0$, the solution $x_{n} \in D_{\varepsilon}, n>0$;

(ii) Attractive if there is $\delta>0$ such that $D\left(x_{i}, \widehat{0}\right)<\delta, i=-k,-k+1, \ldots, 0$, and one has

$$
\lim _{n \longrightarrow \infty} D\left(x_{n}, \widehat{0}\right)=0
$$

(iii) Asymptotically stable if (i) and (ii) hold simultaneously.

Theorem 7. If the parameters $A, B, C_{i}$ are the positive trivial fuzzy numbers, i.e., positive real numbers, and the initial conditions are the positive fuzzy numbers with $\left[x_{i}\right]_{\alpha} \subset(0, B / 2 A), i=-k,-k+1, \ldots, 0, \alpha \in(0,1]$, then the trivial solution $x=\widehat{0}$ of the fuzzy difference equation (8) is asymptotically stable with respect to $D$ as $n \longrightarrow \infty$.

Proof. The result follows from Theorem 2 and Theorem 6.

\section{Numerical Simulation}

Numerical examples are given in this section to confirm the results of the previous sections and to support the theoretical discussion. The example gives the asymptotic behavior of the solution of the fuzzy difference system (8). The following fuzzy difference equation is considered. 
Example 1. Let $k=5$, and the following fuzzy difference equation is considered:

$$
x_{n+1}=\frac{A x_{n-1} x_{n-2}}{B+\sum_{i=3}^{5} C_{i} x_{n-i}}, \quad n=0,1,2, \ldots,
$$

where $A, B, C_{i}(i=3,4,5)$ are the positive real numbers. By Theorem 7, $[A]_{\alpha}=[A, A]=0.2,[B]_{\alpha}=[B, B]=16,\left[C_{3}\right]_{\alpha}=$ $[C, C]=2,\left[C_{4}\right]_{\alpha}=[C, C]=2,\left[C_{5}\right]_{\alpha}=[C, C]=3, \alpha \in(0,1]$. In addition, from Theorem 7 , the initial conditions $x_{-5}, x_{-4}, x_{-3}, x_{-2}, x_{-1}, x_{0}$ with $\left[x_{i}\right]_{\alpha} \subset(0, B / 2 A), i=-5,-4$, $\ldots, 0, \alpha \in(0,1]$, are denoted such that

$$
\begin{aligned}
& x_{0}(x)= \begin{cases}\frac{1}{2} x-\frac{5}{2}, & 5 \leq x \leq 7, \\
-\frac{1}{5} x+\frac{12}{5}, & 7 \leq x \leq 12,\end{cases} \\
& x_{-1}(x)= \begin{cases}\frac{1}{8} x-\frac{3}{8}, & 3 \leq x \leq 11, \\
-\frac{1}{2} x+\frac{13}{2}, & 11 \leq x \leq 13,\end{cases} \\
& x_{-2}(x)= \begin{cases}\frac{1}{8} x-\frac{1}{8}, & 1 \leq x \leq 9, \\
-\frac{1}{4} x+\frac{13}{4}, & 9 \leq x \leq 13,\end{cases} \\
& x_{-3}(x)= \begin{cases}\frac{1}{6} x-\frac{1}{3}, & 2 \leq x \leq 8, \\
-\frac{1}{4} x+3, & 8 \leq x \leq 12,\end{cases} \\
& x_{-4}(x)= \begin{cases}\frac{1}{5} x-\frac{4}{5}, & 4 \leq x \leq 9, \\
-\frac{1}{5} x+\frac{14}{5}, & 9 \leq x \leq 14,\end{cases} \\
& x_{-5}(x)= \begin{cases}\frac{1}{4} x-\frac{3}{2}, & 6 \leq x \leq 10, \\
-\frac{1}{6} x+\frac{8}{3}, & 10 \leq x \leq 16 .\end{cases}
\end{aligned}
$$

From (61), the corresponding triangular fuzzy numbers are obtained:

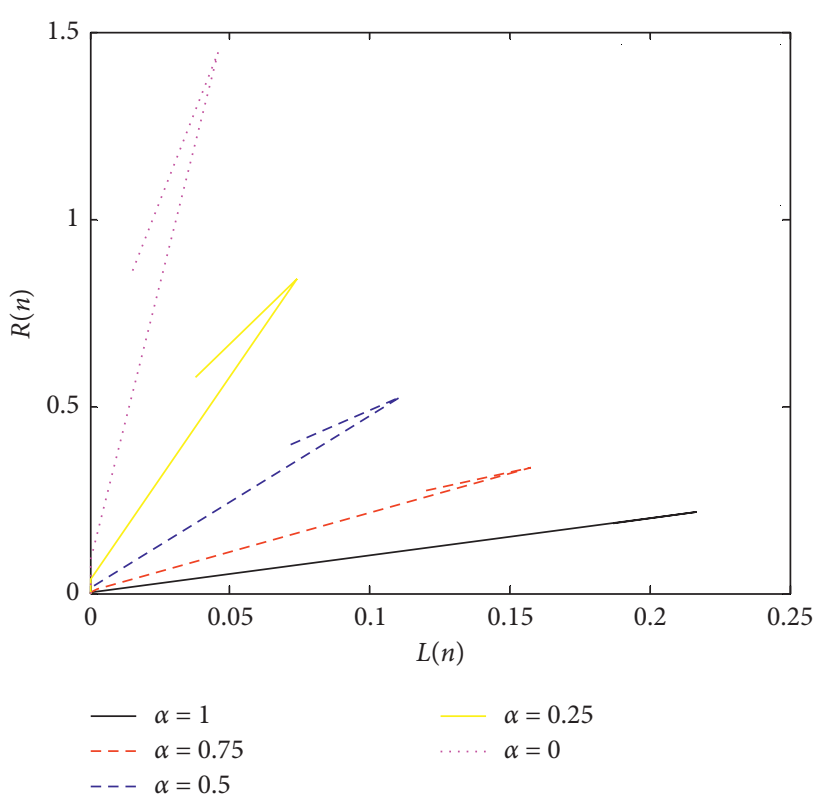

Figure 1: The dynamics of system (63).

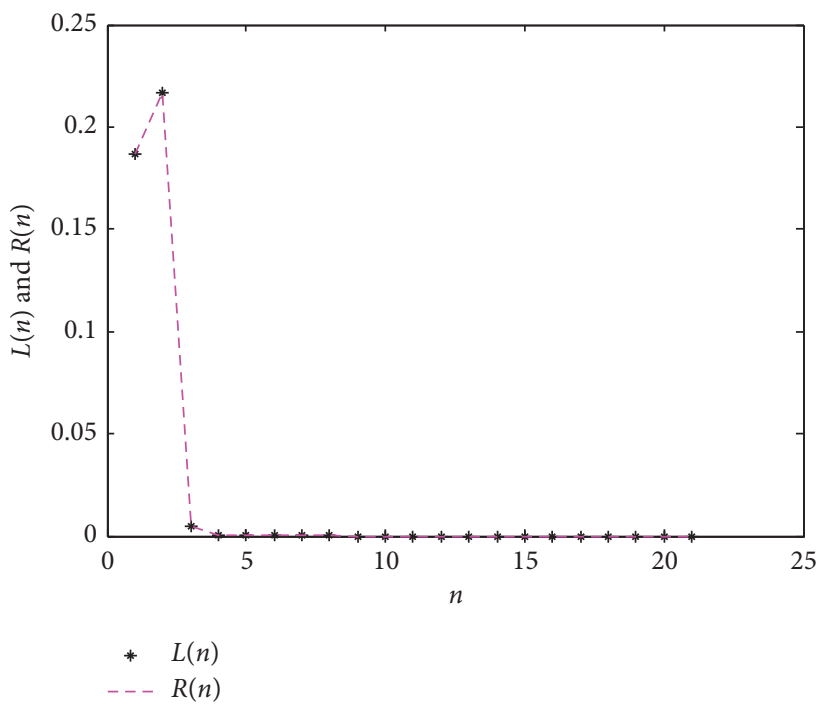

Figure 2: The solution of system (63) when $\alpha=1$.

$$
\begin{aligned}
{\left[x_{0}\right]_{\alpha} } & =[5+2 \alpha, 12-5 \alpha], \\
{\left[x_{-1}\right]_{\alpha} } & =[3+8 \alpha, 13-2 \alpha], \\
{\left[x_{-2}\right]_{\alpha} } & =[1+8 \alpha, 13-4 \alpha], \\
{\left[x_{-3}\right]_{\alpha} } & =[2+6 \alpha, 12-4 \alpha], \\
{\left[x_{-4}\right]_{\alpha} } & =[4+5 \alpha, 14-5 \alpha], \\
{\left[x_{-5}\right]_{\alpha} } & =[6+4 \alpha, 16-6 \alpha] .
\end{aligned}
$$

From (60), the parameters $A, B, C_{i}(i=3,4,5)$ and initial values $x_{-5}, x_{-4}, x_{-3}, x_{-2}, x_{-1}, x_{0}$ satisfy the following system of nonlinear difference equation with parameter $\alpha$ : 


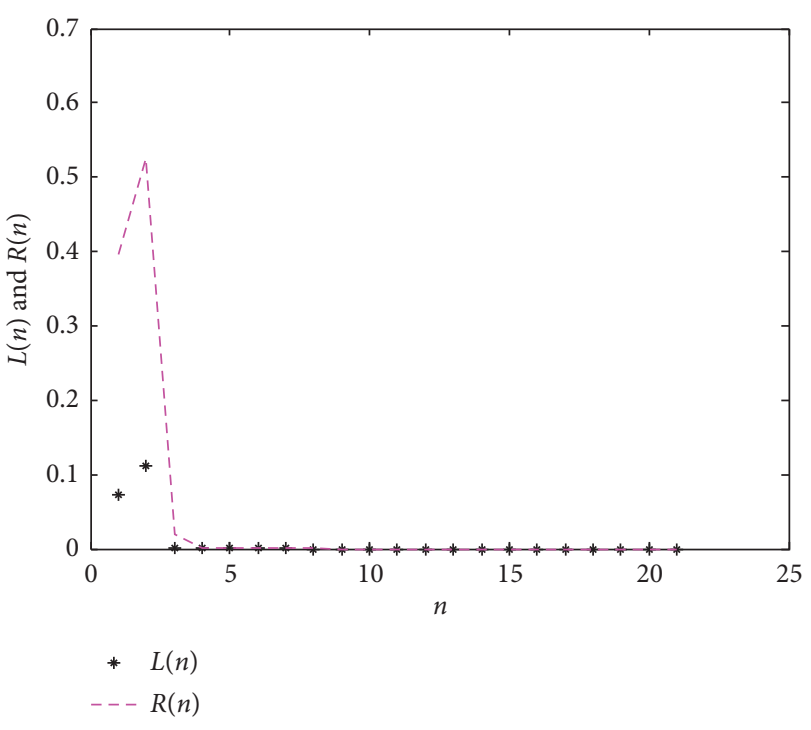

FIgURE 3: The solution of system (63) when $\alpha=0.5$.

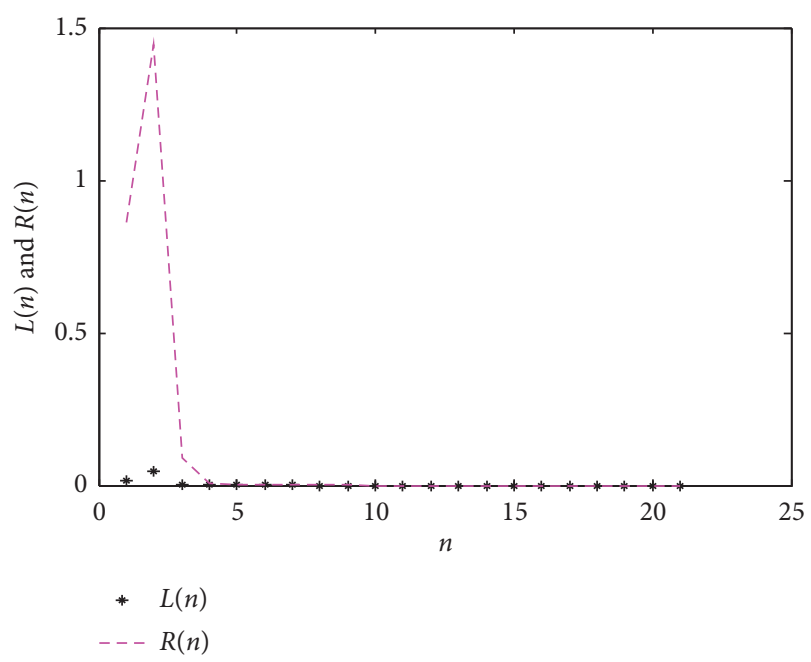

FIgURE 4: The solution of system (63) when $\alpha=0$.

$$
\begin{aligned}
L_{n+1, \alpha} & =\frac{A_{l, \alpha} L_{n-1, \alpha} L_{n-2, \alpha}}{B_{r, \alpha}+\sum_{i=3}^{5} C_{i l, \alpha} R_{n-i, \alpha}}, \\
R_{n+1, \alpha} & =\frac{A_{r, \alpha} R_{n-1, \alpha} R_{n-2, \alpha}}{B_{l, \alpha}+\sum_{i=3}^{5} C_{i l, \alpha} L_{n-i, \alpha}},
\end{aligned}
$$

$$
\alpha \in(0,1], n=0,1, \ldots
$$

It is easy to prove that $\left[x_{i}\right]_{\alpha} \subset(0, B /$ $2 A), i=-5,-4, \ldots, 0$, for $\alpha \in(0,1]$, namely, the conditions of Theorem 7 is satisfied. So from Theorem 7 , the trivial solution $x=\widehat{0}$ of the fuzzy difference (6) is asymptotically stable with respect to $D$ as $n \longrightarrow \infty$ (see Figures 1-4).

\section{Conclusion}

In this paper, a variational iterative method for the nonlinear fuzzy difference equations is proposed. This method is a powerful tool for solving various fuzzy difference equations and can also be applied to other nonlinear differential equations or difference equations in mathematical physics. Numerical simulation results show that this method is effective and simple. Variational iterative method is an effective method to deal with nonlinear structures. MATLAB 2016 software package was used for calculation.

The purpose of this paper was to study the dynamic behavior for a class of nonlinear high-order fuzzy difference equations. Firstly, the existence and uniqueness of positive fuzzy solutions are proved. Secondly, it is obtained that the nonzero equilibrium points of the corresponding ordinary difference equations (32) are unstable by using the linearization method. Finally, it is found that the trivial solution $\widehat{0}$ of fuzzy difference (6) is asymptotically stable when the parameters $A, B, C_{i}$ are positive trivial fuzzy numbers. In particular, an illustrate example is given to show the effectiveness of the obtained results. In addition, the sufficient conditions obtained in this paper are very simple and provide some flexibility for the application and analysis of nonlinear fuzzy difference equations.

\section{Data Availability}

The data used to support the findings of this work are included within this article.

\section{Conflicts of Interest}

The author declares that there are no conflicts of interest.

\section{Acknowledgments}

This work was supported by the Scientific Research Fund of the Yunnan Provincial Education Department of China under grant number 2018JS737 and the Program for Innovative Research Team (in Science and Technology) in University of Yunnan Province.

\section{References}

[1] M. Guo, X. P. Xue, and R. L. Li, "Impulsive functional differential inclusions and fuzzy population models," Fuzzy Sets and Systems, vol. 138, no. 3, pp. 601-615, 2003.

[2] E. Y. Deeba and A. De Korvin, "Analysis by fuzzy difference equations of a model of $\mathrm{CO}_{2}$ level in the blood," Applied Mathematics Letters, vol. 12, no. 3, pp. 33-40, 1999.

[3] E. C. Pielou, Population and Community Ecology: Principles and Methods, CRC Press, London, UK, 1974.

[4] E. P. Popov, Automatic Regulation and Control, Nauka, Moscow, 1966.

[5] K. A. Chrysafis, B. K. Papadopoulos, and G. Papaschinopoulos, "On the fuzzy difference equations of finance," Fuzzy Sets and Systems, vol. 159, no. 24, pp. 3259-3270, 2008.

[6] R. DeVault, G. Ladas, and S. W. Schultz, "On the recursive sequence $x_{n+1}=A / x_{n}+1 / x_{n-2}$," Proceedings of the American Mathematical Society, vol. 126, no. 11, pp. 3257-3262, 1998.

[7] G. Papaschinopoulos and C. J. Schinas, "On a system of two nonlinear difference equations," Journal of Mathematical Analysis and Applications, vol. 219, no. 2, pp. 415-426, 1998. 
[8] Q. L. He, C. Y. Tao, and T. X. Sun, "Periodicity of the positive solutions of a fuzzy max- difference equation," Abstract and Applied Analysis, vol. 2014, Article ID 760247, 4 pages, 2014.

[9] Q. H. Zhang, J. Z. Liu, and Z. G. Luo, "Dynamical behavior of a third-order rational fuzzy difference equation," Advances in Difference Equations, vol. 2015, no. 1, p. 108, 2015.

[10] A. Khastan, "New solutions for first order linear fuzzy difference equations," Journal of Computational and Applied Mathematics, vol. 312, pp. 156-166, 2017.

[11] C. Y. Wang and S. Wang, "Global behavior of equilibrium point for a class of fractional difference equation," in Proceeding of the 7th Asian Control Conference, August 2009, Hong Kong, China.

[12] C. Wang, X. Su, P. Liu, X. Hu, and R. Li, "On the dynamics of a five-order fuzzy difference equation," The Journal of Nonlinear Sciences and Applications, vol. 10, no. 6, pp. 3303-3319, 2017.

[13] X. M. Jia, L. X. Hu, and W. T. Li, "Dynamics of a rational difference equation," Advances in Difference Equations, vol. 2010, Article ID 970720, 15 pages, 2010.

[14] G. Y. Wang and Q. H. Zhang, "Dynamical behavior of firstorder nonlinear fuzzy difference equation," IAENG International Journal of Computer Science, vol. 45, no. 4, pp. 552-559, 2018.

[15] Q. H. Zhang and F. B. Lin, "On dynamical behavior of discrete time fuzzy logistic equation," Discrete Dynamics in Nature and Society, vol. 2018, no. 8, Article ID 8742397, 2018.

[16] E. M. Elsayed and F. Alzahrani, "Periodicity and solutions of some rational difference equations systems," Journal of Applied Analysis and Computation, vol. 9, no. 6, pp. 2358-2380, 2019.

[17] Q. Zhang, F. Lin, X. Zhong et al., "On discrete time BevertonHolt population model with fuzzy environment," Mathematical Biosciences and Engineering, vol. 16, no. 3, pp. 1471$1488,2019$.

[18] Q. Zhang, L. Yang, and D. Liao, “On first order fuzzy Ricatti difference equation," Information Sciences, vol. 270, no. 20, pp. 226-236, 2014.

[19] B. Bede, Mathematics of Fuzzy Sets and Fuzzy logic, Studies in Fuzziness and Soft Computing, Springer, Berlin, Germany, 2013.

[20] P. Diamond and P. Kloeden, Metric Spaces of Fuzzy Sets, Theory and Applications, World Scientific Publishing Co., Inc., River Edge, NJ, USA, 1994.

[21] V. L. Kocic and G. Ladas, Global Behavior of Nonlinear Difference Equations of Higher Order with Applications, Kluwer Academic Publishers Group, Dordrecht, Netherlands, 1993.

[22] H. Sedaghat, Nonlinear Difference Equations: Theory with Applications to Social Science Models, Mathematical Modelling: Theory and Applications, Kluwer Academic Publishers, Dordrecht, Netherlands, 2003.

[23] E. Camouzis and G. Ladas, "Dynamics of third-order rational difference equations with open problems and conjectures," Advances in Discrete Mathematics and Applications, Chapman \& Hall/CRC, Boca Raton, FL, USA, 2008.

[24] G. Papaschinopoulos and B. K. Papadopoulos, "On the fuzzy difference equation $x_{n}+1=A+x_{n} / x_{n}-m$." Fuzzy Sets and Systems, vol. 129, no. 1, pp. 73-81, 2002.

[25] C. Wu and B. Zhang, "Embedding problem of noncompact fuzzy number space E (I)," Fuzzy Sets and Systems, vol. 105, no. 1, pp. 165-169, 1999. 